\title{
The role of the HPA-axis in understanding psychopathology: cause, consequence, mediator, or moderator?
}

\author{
Jan K. Buitelaar
}

Published online: 19 June 2013

(C) Springer-Verlag Berlin Heidelberg 2013

Human life is not without stressful challenges. These challenges may be even needed to fully exploit one's potential, and not only include negative experiences in case of the loss of a loved significant other but also be positively colored, such as moving to a new job and trying to meet high-set expectations. We have been endowed with biobehavioral mechanisms to deal with such challenges. One of these mechanisms is the hypothalamus-pituitary-adrenal (HPA)-axis which is a central component of the body's neuroendocrine response to stress. The hypothalamus secretes corticotropin-releasing hormone (CRH), which in turn stimulates the release of adrenocorticotropic hormone (ACTH) in the anterior pituitary. ACTH in turn stimulates the adrenal cortex to secrete cortisol. Cortisol is known as the major end product of the HPA-axis in humans [1]. Yet, activity of the HPA-axis does not end with the production of cortisol. The HPA-axis is controlled by negative feedback regulation that tends to normalize secretion of cortisol [1]. Elevated cortisol levels reduce ACTH levels as a consequence of negative feedback regulation, followed by a reduction in cortisol levels; in case of reduced cortisol levels, there is less negative feedback regulation at the pituitary, followed by an elevation in ACTH levels [2]. In normal nonstressful situations, cortisol secretion follows a circadian rhythm characterized by high levels in the morning followed by a decrease throughout the rest of the day. Generally, cortisol levels rise in about half an hour after awakening, which is known as the cortisol awakening

J. K. Buitelaar $(\square)$

Department of Cognitive Neuroscience, Radboud University Nijmegen Medical Center, and Karakter Child and Adolescent Psychiatry, P.O. Box 9101, 6500 HB Nijmegen, The Netherlands e-mail: j.buitelaar@psy.umcn.nl response (CAR). The CAR is probably related to the anticipation of the stressfulness of the upcoming day [3].

For several decades the inability to deal with stressful challenges has been considered to be a risk factor at least and a causal factor more likely for various forms of psychopathology. Depressed patients often have a disturbed circadian rhythm of cortisol with overall increased activity of the HPA-axis and increased cortisol levels, and an inability of the HPA-axis to return to normal functioning following a stressor [2]. In fact, depression is currently coined as the stress-related form of psychopathology where the abnormal functioning of the HPA-axis is considered to be linked to the key pathophysiologic mechanisms. Dysregulation of the feedback sensitivity of the HPA-axis established after assessing cortisol levels following a pharmacological challenge with dexamethasone has been documented particularly in about $50 \%$ of patients with major depressive disorder [2]. However, due to the substantial clinical and biological heterogeneity of depression, neither the dexamethasone suppression test nor any of its variant approaches nor any other measures of cortisol have been validated as the biomarker of depression. Its sensitivity and specificity are simply below accepted standards of useful clinical tests. One way to move forward has been to study phenotypically more homogenous dimensions of depression. Adopting this approach in a large sample of depressed patients and controls, nonlinear associations with the shape of an inverted $U$ were found between general distress, anhedonic depression, and anxious arousal on one hand and total morning secretion and the dynamic of the CAR by contrast. Both high and low severity levels were associated with a lower CAR, compared with intermediate levels of severity [4]. Studies in pediatric depression indicate that atypical HPA-axis functioning precedes the emergence of clinical levels of depression and that the 
HPA-axis becomes increasingly dysregulated from child to adult manifestations of depression [5]. Thus, this may explain less strong associations between the HPA-axis and depressive symptoms in children and adolescents, compared to adults, and points to the relevance of taking developmental factors into account.

Abnormal functioning of the HPA-axis has also been implicated in externalizing forms of psychopathology, including aggression and psychopathy. Low HPA-axis activity is associated with low levels of arousal of the central nervous system [6, 7]. According to the stimulationseeking theory, low arousal represents an unpleasant condition which may lead to stimulus-seeking behavior to attain higher and more pleasant levels of arousal [8], which would predispose to externalizing behavior problems. Low basal cortisol levels and low reactivity to challenges was indeed demonstrated for children with clinical oppositional defiant and conduct disorder, see e.g. [9]. However, metaanalysis showed that association between low basal cortisol levels and externalizing behavior problems was, albeit significant, rather weak, whereas cortisol reactivity was not consistently related with externalizing behavior problems [10]. In fact, the relationship between HPA-axis and externalizing behaviors turned out to be much more complex, and moderated by factors as gender, comorbidity with internalizing psychopathology, and perinatal risk factors [11-13].

An integrated analysis of both a large population cohort of adolescents and a clinical cohort of adolescents was able to confirm hypotheses on the association between singleday cortisol (basal morning levels and CAR) and specifically constructed dimensions of anxiety (cognitive versus somatic), depressive (cognitive-affective versus somatic), and externalizing problems (reactive versus proactive aggression), and explored the modifying role of sex. Most support was found for higher cortisol (mainly CAR) in relation to depressive problems. However, in general, associations were weak in both samples [14].

In this issue, Isaksson and colleagues [15] report that cortisol levels were lower in ADHD than in control children, a finding consistent with a recent meta-analysis that established lower baseline cortisol levels in ADHD compared to control subjects with an effect size of 0.3 [16]. A small effect size, but still meaningful. Next, Isaksson et al. attempted to explore whether exposure to fetal and childhood psychosocial adversity could explain these low cortisol levels in ADHD through early programming of the HPA-axis. Children with ADHD had indeed greater exposure to fetal and childhood adversity than comparison children, but this could not explain lower cortisol levels in ADHD patients. Their analysis did not control for comorbid anxiety and depression, but if comorbid anxiety or depression would have been present more often in ADHD than in comparison children, it would just have diminished the group difference in cortisol levels. The authors speculate that genetic factors that are shared between the ADHD phenotype and the HPA-axis functioning may be responsible for the lower cortisol levels in ADHD. This is a hypothesis that can be tested in twin studies that have collected both cortisol data and ratings on ADHD symptoms. If supported, this would suggest that abnormal HPA-axis functioning is on the causal pathway of ADHD rather than being a consequence of exposure to life stress, or a mediator of the relationship between environmental challenges and ADHD. It would fit into findings of low central arousal in ADHD, as reflected in the observation that slow wave activity is increased and fast wave activity is decreased in most children with ADHD [17].

Recently, new theoretical perspectives have been added to the role of the HPA-axis in developmental psychopathology. The evolutionary-developmental theory of biological sensitivity to context (BSC) offers a conceptual framework for understanding individual differences in biological sensitivity to the environment [18]. According to the developmental programming part of this theory, both children who experience high-stress environments in early life, and children who experience supportive, low-stress environments in early life tend to develop a highly reactive stress response system. In addition, children who experience moderate stress environments tend to develop a low reactive stress response system. Although initial formulation of BSC theory mainly seemed to apply to stress reactivity, the developmental programming part of BSC theory has recently been described in much greater detail, now also involving basal cortisol levels [19]. These theories would mean, to put it simply, that whether a certain baseline level and responsivity of the HPA-axis would be optimal, suboptimal or even detrimental depends on the characteristics of the environment in which the person is living.

How far are we in analyzing the role of the HPA-axis in developmental psychopathology, and in which direction should research move?

1. Associations such as between increased cortisol levels and depression and anxiety, and between low levels and externalizing behaviors are obviously stronger for clinical and/or persisting cases than for population samples, and thus for lower symptom levels. Effects at the population level are rather weak and often inconsistent.

2. The associations appear to be somewhat stronger for specific symptom dimensions (cognitive-affective versus somatic symptoms of depression; instrumental versus affective aggression) than for DSM categories.

3. The associations appear to be co-dependent on gender, and of course, comorbidity. 
4. The mechanisms underlying these associations in clinical cases appear to domain-specific. The dysregulation of the HPA-axis in depression has been linked to the possible neurotoxic influence of the increased levels of cortisol on the hippocampus, affecting neuronal plasticity. In contrast, the role in externalizing disorders is more likely to be put into the perspective of low arousal and hence insufficient ability to mobilize resources dealing with just normal and more extreme challenges.

5. Research so far hardly addresses the issue whether dysregulated HPA-axis functioning is a consequence rather than a causal or contributing factor to psychopathology. Being hyperactive, impulsive and inattentive leads more likely to impairment of functioning, life stress, negative feedback from the environment, etc. These experiences will tend to factor in, and impact systems of stress regulation.

6. So far, the role of the environment has been poorly explored. Most studies that speak about the environment focus on severe life events and trauma, but much less about normal variation in stressful challenges, and even about the evolutionary perspective and early programming effects. In this vein, one would like to see studies on the stress system's contribution to mediate or moderate effects of stressful challenges.

7. Prospective longitudinal studies about the stability and change of the HPA-axis functioning are rare, if any, and need to be performed more systematically.

8. Assessment of the HPA-axis functioning should be more thorough and move beyond single-day cortisol measurements to include reactions to standard stimuli, and take into account the role of other substances that modulate the stress axis, such as vasopressin, oxytocin, androgenic hormones and steroids [20].

\section{References}

1. Tsigos C, Chrousos GP (2002) Hypothalamic-pituitary-adrenal axis, neuroendocrine factors and stress. J Psychosom Res 53(4):865-871

2. Gold PW, Drevets WC, Charney DS (2002) New insights into the role of cortisol and the glucocorticoid receptor in severe depression. Biol Psychiatry 52(5):381-385

3. Fries E, Dettenborn L, Kirschbaum C (2009) The cortisol awakening response (CAR): facts and future directions. Int $\mathbf{J}$ Psychophysiol 72(1):67-73

4. Wardenaar KJ, Vreeburg SA, van Veen T, Giltay EJ, Veen G, Penninx BW, Zitman FG (2011) Dimensions of depression and anxiety and the hypothalamo-pituitary-adrenal axis. Biol Psychiatry 69(4):366-373

5. Guerry JD, Hastings PD (2011) In search of HPA axis dysregulation in child and adolescent depression. Clin Child Fam Psychol Rev 14(2):135-160
6. Chrousos GP, Gold PW (1998) A healthy body in a healthy mind-and vice versa-the damaging power of "uncontrollable" stress. J Clin Endocrinol Metab 83(6):1842-1845

7. van Goozen SH, Matthys W, Cohen-Kettenis PT, Buitelaar JK, van Engeland H (2000) Hypothalamic-pituitary-adrenal axis and autonomic nervous system activity in disruptive children and matched controls. J Am Acad Child Adolesc Psychiatry 39(11): $1438-1445$

8. Raine A (1996) Autonomic nervous system factors underlying disinhibited, antisocial, and violent behavior. Biosocial perspectives and treatment implications. Ann N Y Acad Sci 20(794): 46-59

9. Popma A, Doreleijers TA, Jansen LM, Van Goozen SH, Van Engeland H, Vermeiren R (2007) The diurnal cortisol cycle in delinquent male adolescents and normal controls. Neuropsychopharmacology 32(7):1622-1628

10. Alink LR, van Ijzendoorn MH, Bakermans-Kranenburg MJ, Mesman J, Juffer F, Koot HM (2008) Cortisol and externalizing behavior in children and adolescents: mixed meta-analytic evidence for the inverse relation of basal cortisol and cortisol reactivity with externalizing behavior. Dev Psychobiol 50(5): $427-450$

11. Marsman R, Rosmalen JG, Oldehinkel AJ, Ormel J, Buitelaar JK (2009) Does HPA-axis activity mediate the relationship between obstetric complications and externalizing behavior problems? The TRAILS study. Eur Child Adolesc Psychiatry 18(9):565-573

12. Marsman R, Nederhof E, Rosmalen JG, Oldehinkel AJ, Ormel J, Buitelaar JK (2012) Family environment is associated with HPAaxis activity in adolescents. The TRAILS study. Biol Psychol 89(2):460-466

13. Marsman R, Swinkels SH, Rosmalen JG, Oldehinkel AJ, Ormel J, Buitelaar JK (2009) HPA-axis activity and externalizing behavior problems in early adolescents from the general population: the role of comorbidity and gender. The TRAILS study. Psychoneuroendocrinology 33(6):789-798

14. Dietrich A, Ormel J, Buitelaar JK, Verhulst FC, Hoekstra PJ, Hartman CA (2012). Cortisol in the morning and dimensions of anxiety, depression, and aggression in children from a general population and clinic-referred cohort: an integrated analysis. The TRAILS study. Psychoneuroendocrinology Dec 10 [Epub ahead of print]

15. Isaksson J, Nilsson KW, Lindblad, F (2013). Early psychosocial adversity and cortisol levels in children with attention-deficit/ hyperactivity disorder. Eur Child Adolesc Psychiatry (this issue)

16. Scassellati C, Bonvicini C, Faraone SV, Gennarelli M (2012) Biomarkers and attention-deficit/hyperactivity disorder: a systematic review and meta-analyses. J Am Acad Child Adolesc Psychiatry 51(10):1003-1019

17. Barry RJ, Clarke AR, Johnstone SJ (2003) A review of electrophysiology in attention-deficit/hyperactivity disorder: I. Qualitative and quantitative electroencephalography. Clin Neurophysiol 114(2):171-183

18. Boyce WT, Ellis BJ (2005) Biological sensitivity to context: I. An evolutionary-developmental theory of the origins and functions of stress reactivity. Dev Psychopathol 17(2):271-301

19. Del Giudice M, Ellis BJ, Shirtcliff EA (2011) The adaptive calibration model of stress responsivity. Neurosci Biobehav Rev 35(7):1562-1592

20. Swaab DF, Bao AM, Lucassen PJ (2005) The stress system in the human brain in depression and neurodegeneration. Ageing Res Rev 4(2):141-194 